\title{
The Relationship Between Patient-Centeredness and Cultural Competence Among Primary Care Physicians in the Southern US
}

J Gen Intern Med 36(10):3252-3

DOI: $10.1007 / \mathrm{s} 11606-020-06298-9$

(c) Society of General Internal Medicine 2020

\section{INTRODUCTION}

Both patient-centeredness (PC) and cultural competence (CC) could reduce health care inequities. ${ }^{1,2}$ While distinct concepts, they are related, ${ }^{1,3}$ though there is no research exploring their correlation among primary care providers. In order to advance health equity training among health care providers, it is important to examine the correlation between the two concepts. It was hypothesized that there would be a significant relationship between PC and CC among primary care physicians (PCPs); there would be a stronger correlation between $\mathrm{PC}$ (caring) and $\mathrm{CC}$ than for PC (sharing) and $\mathrm{CC}$; and there would be a relationship between demographic variables and $\mathrm{PC} / \mathrm{CC}$ variables.

\section{METHODS}

Following Morehouse School of Medicine Institutional Review Board approval, a convenience sample of 92 PCPs (family medicine, internal medicine) was recruited in late 2012-early 2013 in the Southern US region. An online questionnaire was distributed to PCPs to test the above-mentioned hypotheses.

PC was measured using the 18-item self-report PatientPractitioner Orientation Scale (PPOS), consisting of two subscales: (1) sharing (shared decision-making/information sharing) and (2) caring (discussion of psychosocial issues in medical consultations, treating the patient as a whole person). ${ }^{4}$ $\mathrm{CC}$ was assessed using the 25-item self-report Cultural Competence Assessment (CCA), also including two subscales: (1) CC awareness/sensitivity and (2) CC behaviors. ${ }^{5}$

At the time the research was conducted, Dr. Xanthos was an Assistant Professor at Morehouse School of Medicine.

Prior Presentations The preliminary results of this study were presented as a poster at the Morehouse College Health Disparities Symposium, April 17, 2013, Atlanta, GA.

Received June 25, 2020

Accepted October 5, 2020

Published online October13, 2020
Descriptive statistics were computed for the variables of interest; Pearson's correlation analysis was used to test the strength of the association between PPOS and CCA scores; and the scores were assessed for a relationship with the demographics (years since medical school graduation, gender, cultural identity, and previous cultural competence training) using a one-way ANOVA (PASW Statistics 18, SPSS).

\section{RESULTS}

The sample consisted of 52 (56.5\%) women and 40 (43.5\%) men; 58 (63.7\%) were White, $18(19.8 \%)$ were Black/African-American, $13(14.3 \%)$ were Asian, and 2 (2.2\%) identified with more than one group (Table 1). The average number of years since graduation from medical school was 20.37 and $83.7 \%$ of PCPs (77 participants) had participated in CC training (Table 1). The mean PPOS score (patient-centeredness) was 4.88 (highest possible score is 6) and the mean CCA score (cultural competence) was 3.75 (highest possible score is 5).

There was a moderate correlation between $\mathrm{PC}$ and $\mathrm{CC}$ scores among the PCPs $(r=0.403, p<0.0005)$. The "caring" element of PC was more closely associated with CC $(r=$ $0.417, p<0.0005)$ than the "sharing" component of PC $(r=$ $0.277, p=0.007)$. There was a significant relationship between CC scores and CC training $(F(1,77)=4.65, p=0.034)$; however, the other demographic covariates did not show an association with $\mathrm{PC} / \mathrm{CC}$ scores.

Table 1 Demographic Characteristics of PCPs

\begin{tabular}{ll}
\hline \hline Variables & $\mathbf{N = 9 2}$ \\
\hline Years since graduation from medical school, mean & 20.37 \\
(SD) & $(11.17)$ \\
Gender, $n(\%)$ & $40(43.5)$ \\
$\quad$ Male & $52(56.5)$ \\
Female & \\
Cultural identity*, $n(\%)$ & $58(63.7)$ \\
White & $18(19.8)$ \\
Black/African-American & $13(14.3)$ \\
Asian & $2(2.2)$ \\
From multiple races & \\
Cultural competence training & $\dagger, n(\%)$ \\
Yes & $77(83.7)$ \\
No & $14(15.2)$ \\
\hline
\end{tabular}

*There was one missing response for this question

tThere was one missing response for this question 


\section{DISCUSSION}

This is the first study to explore the relationship between PC and $\mathrm{CC}$ among PCPs, and confirms a modest correlation between the two. The finding that $\mathrm{CC}$ was less correlated with PC (sharing) than PC (caring) suggests that CC may be less associated with shared decision-making than with the other aspects of PC. This is supported by the literature ${ }^{3,6}$ which indicates that $\mathrm{CC}$ does not address the sharing of power in the physician-patient relationship (though this is a core component of PC); rather, academic commentary suggests that $\mathrm{CC}$ focuses on the acquisition of knowledge about cultural groups. ${ }^{3,6}$ In short, the literature and study findings indicate that while shared decision-making is a key element of PC, it may not be a universal feature of CC.

Some limitations of the study should be noted. First, the sample size of physicians was relatively small; future research would benefit from a factor analysis of the questions from the PPOS and CCA, using a larger sample. Second, the potential for self-selection bias among the study participants was another limitation; it is possible that physicians who were more patient-centered/culturally competent were more likely to participate in the survey. Finally, participant responses were selfreported, and therefore, it is difficult to ascertain how well these scores reflect the providers' actual behavior and skills.

In order to promote health equity, there is a need for further study of PC and CC concepts, training programs, and curricula. Quantitative and qualitative methods should be utilized to further clarify the two constructs; identify best practices; and determine the effectiveness of training programs on improving health outcomes among diverse populations.

Acknowledgments: The author wishes to thank all the primary care physicians who participated in this study. The author is also grateful to Kevin Fiscella, $M D$, Robert Like, $M D$, and Susan Shapiro, $P h D, R N$, for their helpful comments on earlier versions of this work.
Clare Xanthos, $\mathrm{PhD}, \mathrm{MSc}^{1,2}$

${ }^{1}$ Department of Community Health and Preventive Medicine, Morehouse School of Medicine,

Atlanta, GA, USA

${ }^{2}$ Barnet, UK

Corresponding Author: Clare Xanthos, PhD, MSc; Barnet, UK (e-mail: clare.xanthos@gmail.com).

Funding This work was supported by the Clinical Research Education Career Development (CRECD) in Minority Institutions Program, NIH Grant \#: R25 RRO17694.

\section{Compliance with Ethical Standards:}

Conflict of Interest: The author declares that she does not have a conflict of interest.

\section{REFERENCES}

1. Saha S, Beach MC, Cooper LA. Patient centeredness, cultural competence and healthcare quality. J Natl Med Assoc 2008;100:1275-1285.

2. Ignatius Bau JD, Logan RA, Dezii C, Rosof B, Fernandez A. PatientCentered, Integrated Health Care Quality Measures Could Improve Health Literacy, Language Access, and Cultural Competence. Washington: National Academy of Medicine; 2019.

3. Stubbe DE. Practicing cultural competence and cultural humility in the care of diverse patients. Focus (Am Psychiatr Publ) 2020;18(1):49-51.

4. Krupat E, Rosenkranz SL, Yeager CM, Barnard K, Putnam SM, Inui TS. The practice orientations of physicians and patients: the effect of doctorpatient congruence on satisfaction. Patient Educ Couns 2000;39(1):49-59.

5. Schim S, Doorenbos A, Miller J, Benkert R. Development of a cultural competence assessment instrument. J Nurs Meas 2003;11(1):29-40.

6. Tervalon M, Murray-García J. Cultural humility versus cultural competence: a critical distinction in defining physician training outcomes in multicultural education. J Health Care Poor Underserved 1998;9(2):117125.

Publisher's Note: Springer Nature remains neutral with regard to jurisdictional claims in published maps and institutional affiliations. 